\title{
Non-Invasive Detection of Intracranial Hypertension Using Random Forests
}

\author{
Federico Wadehn ${ }^{1}$, Dario Walser ${ }^{1}$, Michal Bohdanowicz ${ }^{2}$, Marek Czosnyka ${ }^{3}$, Thomas Heldt ${ }^{4}$ \\ ${ }^{1}$ Signal and Information Processing Laboratory, ETH Zurich, Switzerland \\ ${ }^{2}$ Warsaw University of Technology, Poland, ${ }^{3}$ University of Cambridge, Cambridge, UK \\ ${ }^{4}$ Massachusetts Institute of Technology, Cambridge, MA, USA
}

\begin{abstract}
In various pathologies, such as hemorrhagic stroke or traumatic brain injury, intracranial pressure (ICP) can rise to the point of causing neurological damage and should consequently be monitored. The invasiveness of current ICP monitoring procedures, however, limits the patient pool. Having a non-invasive screening tool for detecting intracranial hypertension would therefore be of high value. In this work, we developed a binary classifier to determine if ICP is above the clinical cutoff value of 20 mmHg. Arterial blood pressure (ABP) and cerebral blood flow velocity $(C B F V)$ recordings serve as the only inputs to the classifier. We identified eighteen $A B P$ and $C B F V$ features reported to correlate with ICP. Given around $32 \mathrm{~h}$ of $A B P, C B F V$ and invasive ICP recordings from 36 traumatic brain injury patients, we trained different binary classifiers via leave-one-patient-out cross validation. The random forest classifier resulted in the most stable and accurate prediction, yielding a sensitivity of $69.1 \%$ and specificity of $78.3 \%$. These encouraging results and the ease by which features can be added to the framework, suggest possible extensions to include, for example, features derived from venous pressure or near-infrared spectroscopy recordings.
\end{abstract}

\section{INTRODUCTION}

Intracranial pressure (ICP) is an essential neurological parameter that can be elevated in various pathological conditions, such as hydrocephalus, subarachnoid hemorrhage and traumatic brain injury [1]. An increased ICP can lead to brain ischemia, neurological damage and ultimately to brain herniation and death. The clinical gold standard of ICP monitoring involves inserting a pressure probe into the brain tissue or cerebrospinal fluid space. Due to the invasiveness of the procedure, ICP monitoring is often limited to severely ill patients. Several attempts have been made to estimate ICP from clinically available non- or minimally-invasive measurements [2]. Most reviews conclude, however, that current methods to determine ICP non-invasively, especially "ICP as a number" [3] are not sufficiently accurate for clinical practice [2]. Reasons for the difficulty in estimating ICP non-invasively are among others, the large patient variability, the heterogeneity of cerebral blood flow due to autoregulation and the presence of confounding factors such as (central) venous pressure and the partial pressure of oxygen and carbon dioxide [4].

For triage and screening, the exact ICP value might not be needed and knowing if a patient has intracranial hypertension might be sufficient to direct care. Therefore, simplifying the matter, our aim was to classify on a beat-bybeat basis if ICP is above $20 \mathrm{mmHg}$, which is a common clinical cutoff before (aggressive) treatment is initiated [5]. To achieve this, we use two commonly available physiological signals, that influence and are influenced by ICP, namely arterial blood pressure (ABP) and cerebral blood flow velocity (CBFV). These physiological vital signs are routinely monitored in most intensive care units. Basing a classification algorithm on these two signals would therefore not alter clinical practice. ABP is commonly recorded minimally-invasively via a radial artery catheter and CBFV (in the middle cerebral artery) is measured non-invasively using transcranial Doppler ultrasonography. Through a literature search we identified eighteen ABP and CBFV features, that are thought to correlate with ICP. Using these features we trained different supervised learning models: a linear support vector machine (SVM), a Naive Bayes (NB) and a random forest (RF) classifier for detecting an ICP above $20 \mathrm{mmHg}$.

\section{BINARY CLASSIFICATION OF ICP}

To find features that are correlated with ICP, we performed a literature search using PubMed and Google Scholar akin to [3], using the keywords "non-invasive intracranial pressure transcranial Doppler" and variations of it. Thereby, we identified fourteen features for which there is cumulative evidence of moderate to high correlation with ICP $[3,4,6]$. We included four additional features based on physiological considerations, yielding a total of eighteen features. These features are then computed on a beat-by-beat basis, which requires only beat-by- 
beat aligned $\mathrm{ABP}$ and $\mathrm{CBFV}$ waveforms. The exact intrabeat temporal relation between $\mathrm{ABP}$ and $\mathrm{CBFV}$, which are recorded at different sites (radial artery vs middle cerebral artery) and with different devices, is therefore not required. The computed features can be grouped as follows:

ABP features (diastolic, mean and systolic):

$$
\left(\mathrm{ABP}_{\text {dia }}, \mathrm{ABP}_{\text {mean }}, \mathrm{ABP}_{\text {sys }}\right) \text {. }
$$

At highly elevated ICP, brain ischemia triggers the Cushing reflex [7] in which the body attempts to restore adequate cerebral blood flow (CBF) by increasing arterial blood pressure. An increase in systemic blood pressure has also been observed at moderately increased ICP, which causes a change in sympathetic tone [6].

CBFV features (diastolic, mean and systolic):

$$
\left(\mathrm{CBFV}_{\text {dia }}, \mathrm{CBFV}_{\text {mean }}, \mathrm{CBFV}_{\text {sys }}\right) \text {. }
$$

In [8] it was observed that an increase in ICP resulted in a drop in diastolic CBFV. Elevations in ICP lead to a reduction in cerebral perfusion pressure (CPP). When cerebral autoregulatory capacity has been exhausted, such reductions in CPP will reduce cerebral blood flow $(\mathrm{CBF})$. Due to the fairly constant diameter of the middle cerebral artery, a reduction in $\mathrm{CBF}$, will in turn lead to a proportional reduction in CBFV. It is important to note that measured CBFV depends on the angle of insonation of the transcranial Doppler beam. This influence is usually small since the ultrasound beam is practically collinear to the flow direction, when the middle cerebral artery is insonated. To be independent of the angle of insonation, we additionally use ratio of CBFV features: Pulsatility index (PI), resistivity index (RI) and the Systolic-Diastolic Ratio (SDR):

$$
\begin{aligned}
\mathrm{PI}_{\mathrm{CBFV}} & =\left(\mathrm{CBFV}_{\text {sys }}-\mathrm{CBFV}_{\text {dia }}\right) / \mathrm{CBFV}_{\text {mean }} \\
\mathrm{RI}_{\mathrm{CBFV}} & =\left(\mathrm{CBFV}_{\text {sys }}-\mathrm{CBFV}_{\text {dia }}\right) / \mathrm{CBFV}_{\text {sys }} \\
\mathrm{SDR}_{\mathrm{CBFV}} & =\mathrm{CBFV}_{\text {sys }} / \mathrm{CBFV}_{\text {dia }} .
\end{aligned}
$$

The $\mathrm{PI}_{\mathrm{CBFV}}$ has been reported in several works to be positively correlated with ICP (to varying degrees though [3]). In complete analogy, we included the following ABP ratios:

$$
\begin{aligned}
\mathrm{PI}_{\mathrm{ABP}} & =\left(\mathrm{ABP}_{\text {sys }}-\mathrm{ABP}_{\text {dia }}\right) / \mathrm{ABP}_{\text {mean }} \\
\mathrm{RI}_{\mathrm{ABP}} & =\left(\mathrm{ABP}_{\text {sys }}-\mathrm{ABP}_{\text {dia }}\right) / \mathrm{ABP}_{\text {sys }} \\
\mathrm{SD}_{\mathrm{ABP}} & =\mathrm{ABP}_{\text {sys }} / \mathrm{ABP}_{\text {dia }} .
\end{aligned}
$$

We introduced these features for two reasons: Firstly, due to the reported widening of $\mathrm{ABP}$ pulse pressure during the Cushing reflex and in ICP induced arterial hypertension [7]. Secondly, due to the influence of ABP pulsatility (driver of blood flow) on CBFV pulsatility. ABP pulsatility is thus a confounding factor when looking at $\mathrm{PI}_{\mathrm{CBFV}}$ only.
We introduced the following ratios of the aforementioned features:

$$
\begin{aligned}
\mathrm{PI}_{\text {Ratio }} & =\mathrm{PI}_{\mathrm{CBFV}} / \mathrm{PI}_{\mathrm{ABP}} \\
\mathrm{RI}_{\text {Ratio }} & =\mathrm{RI}_{\mathrm{CBFV}} / \mathrm{RI}_{\mathrm{ABP}}
\end{aligned}
$$

Additionally, we included:

$$
\begin{aligned}
\mathrm{PI}_{\mathrm{CBFV}-\mathrm{LR}} & =\left|\mathrm{PI}_{\mathrm{CBFV} \text {-left }}-\mathrm{PI}_{\mathrm{CBFV} \text {-right }}\right| \\
\mathrm{RHO}_{\mathrm{ABP}-\mathrm{CBFV}} & =\rho\left(\mathrm{ABP}_{\text {pulse }}, \mathrm{CBFV}_{\text {pulse }}\right),
\end{aligned}
$$

where $\mathrm{PI}_{\mathrm{CBFV}-\mathrm{LR}}$ measures the asymmetry in pulsatility of CBFV between left and right cerebral artery pair. The correlation coefficient $\mathrm{RHO}_{\mathrm{ABP}-\mathrm{CBFV}}$ between the onsetaligned $\mathrm{ABP}$ and $\mathrm{CBFV}$ waveform pulses assesses similarity between the two and thus the degree of resistive behavior. Due to reported cardiac arrhythmia during intracranial hypertension [9], the heart rate (interbeat interval) was included in the feature vector. Finally, we also use the critical closing pressure [3] as a feature. The critical closing pressure denotes the lower limit of arterial blood pressure below which, due to active wall tension, arterioles collapse and blood flow ceases.

\subsection{Predicting Intracranial Hypertension}

The algorithm can be subdivided into the following parts: Beat detection, signal quality assessment, CBFV channel selection, feature computation, and finally, binary classification of intracranial hypertension.

Beat Detection: Given the ABP and CBFV signals (left and right middle cerebral artery) we perform a beat onset detection using the algorithm presented in [10]. Due to the different ranges of $\mathrm{CBFV}$ signals compared to $\mathrm{ABP}$, we rescale the CBFV signals to the range of $A B P$ to be able to use the onset detection algorithm [10]. The ABP and CBFV beat onsets are then matched to ensure that the features extracted from $\mathrm{ABP}$ and $\mathrm{CBFV}$ pulses belong to the same beat.

Signal Quality and Feature Computation: Following this, we perform a beat-by-beat binary signal quality assessment (SQA) to remove obvious artifacts. In our simple rule-based SQA, the following physiological sanity checks are performed for each beat: $\min (\mathrm{ABP})>30 \mathrm{mmHg}$, $\max (\mathrm{ABP})<300 \mathrm{mmHg}, \max (\mathrm{ABP})-\min (\mathrm{ABP})>$ $20 \mathrm{mmHg}, \min (\mathrm{CBFV})>5 \mathrm{~cm} / \mathrm{s}, \max (\mathrm{CBFV})<$ $300 \mathrm{~cm} / \mathrm{s}$, and beat duration $T_{b}>0.25 \mathrm{~s}$ and $T_{b}<2.5 \mathrm{~s}$. More elaborate template-based SQA algorithms such as [11] can be used when the signal quality is lower. The CBFV channel with the higher percentage of good beats over the entire recording is then chosen for computing the features, except for $\mathrm{PI}_{\mathrm{CBFV}-\mathrm{LR}}$, for which both $\mathrm{CBFV}$ channels are required. For each of the remaining (good) beats, we then compute the aforementioned eighteen features. 
Binary data labels (ground truth) for training and testing are generated by thresholding the beat-by-beat ICP mean value using the common clinical cutoff of $20 \mathrm{mmHg}$. Features and labels are averaged over 8 beats to increase robustness.

Training the Binary Classifiers: We trained and evaluated linear SVM, NB and RF classifiers [12] using leaveone-patient out cross-validation. Training and testing was performed using the machine learning toolbox of MATLAB 2017a (The Mathworks, Inc., USA). A RF classifier is an ensemble method based on building multiple decision trees with a randomized learning procedure. Each decision tree of the RF is built using a training set obtained by sampling from the original training set (bagging). During learning, at each node of the decision tree on which the next split is learned (see Fig. 1), a subset of the eighteen features, in our case eight, is chosen at random. This procedure performs an implicit feature selection and thus reduces the chance of overfitting [12]. We used an ensemble of 256 decision trees and limited their depth to 8 splits. The standard random forest implementation grows unconstrained decision trees. In our implementation this would lead to severe overfitting. This is due to the training samples not being independent, when belonging to the same patient.

The NB and the SVM classifiers are more sensitive to highly correlated (e.g., the pulsatility and resistivity indices) or potentially irrelevant features. Therefore, we resort to an explicit feature ranking and selection step for these classifiers. Based on their correlation with ICP, we used the following seven features: $\mathrm{ABP}_{\text {mean }}, \mathrm{ABP}_{\text {sys }}$, $\mathrm{CBFV}_{\text {dia }}, \mathrm{RI}_{\mathrm{ABP}}, \mathrm{PI}_{\mathrm{CBFV}}, \mathrm{PI}_{\mathrm{CBFV}-\mathrm{LR}}, \mathrm{RHO}_{\mathrm{ABP}-\mathrm{CBFV}}$. For both the NB and SVM we use the plain vanilla MATLAB implementation, except for setting the class prior probabilities for the NB classifier to uniform, to avoid learning the class imbalance. When training the classifiers we gave false negatives (missed intracranial hypertension) a four times higher penalty than to false positives.

\section{Dataset and Results}

Dataset: To train and test our classification approach we used the dataset in [13]. In total we had access to recordings from 36 patients who suffered from traumatic brain injury and were therefore admitted to the neuro-ICU at Addenbroke's Hospital at University of Cambridge UK, in the period 1992-1997. The usage of the anonymized data was approved by the neurocritical care user's committee of the University of Cambridge for subsequent analysis and publications. The database contains $32.4 \mathrm{~h}$ of simultaneous recordings of radial $\mathrm{ABP}, \mathrm{CBFV}$ measured from the left and right middle cerebral artery and invasive ICP sampled at a rate between $20 \mathrm{~Hz}$ and $70 \mathrm{~Hz}$. All signals were upsampled to the common rate of $125 \mathrm{~Hz}$. The SQA step

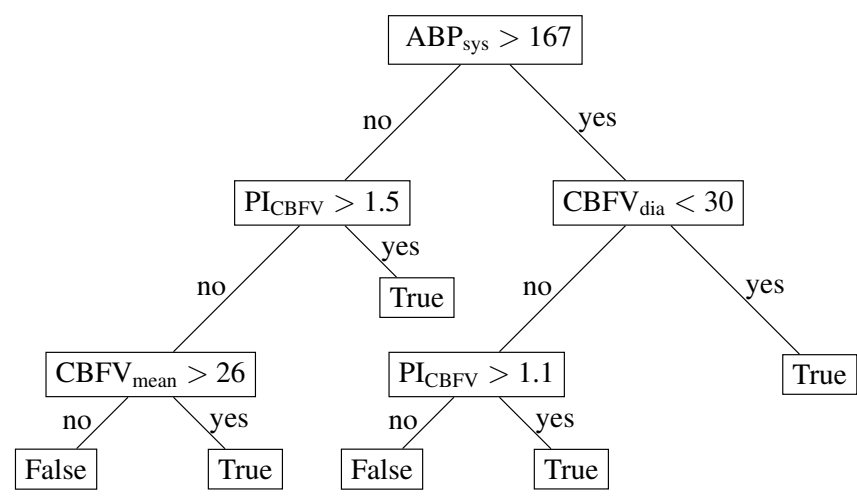

Figure 1. Instance of a decision tree trained by withholding one subject. Class label 'True' refers to ICP> $20 \mathrm{mmHg}$. The tree shown is a pruned version (for display purposes) of the deeper tree that was learned. Units: ABP $[\mathrm{mmHg}], \mathrm{CBFV}[\mathrm{cm} / \mathrm{s}]$ and the ratios are dimensionless.

discarded only $7 \%$ of the beats, mainly due to artifacts in the CBFV signal. It is worth noting that nowadays, due to the strict treatment protocols, it is quite rare to have ICP recordings over such large ranges (up to $75 \mathrm{mmHg}$ ), which makes this a highly valuable database.

Results: The RF classifier achieved the best performance in terms of sensitivity (69.1\%) and had a comparable specificity $(78.3 \%)$ to the other classifiers. Tables 1 and 2 show the respective confusion matrices on the training and test data for the RF. Both the linear SVM (sensitivity $56.2 \%$ and specificity $77.4 \%$ ) and the NB classifier (sensitivity $54.4 \%$ and specificity $79.1 \%$ ) were outperformed by the RF in terms of sensitivity, which is the more relevant statistical measure when screening patients.

$\begin{array}{lccc} & \text { Pred. Pos. } & \text { Pred. Neg } & \\ \text { Cond. Pos. } & 28.4 \% & 4.0 \% & \text { SES }=87.7 \% \\ \text { Cond. Neg. } & 12.1 \% & 55.5 \% & \text { SPC }=82.1 \%\end{array}$

Table 1. Averaged confusion matrix of RF-based classification on the training sets along with sensitivity (SES) and specificity (SPC).

$\begin{array}{lccc} & \text { Pred. Pos. } & \text { Pred. Neg } & \\ \text { Cond. Pos. } & 22.4 \% & 10.0 \% & \text { SES }=69.1 \% \\ \text { Cond. Neg. } & 14.7 \% & 52.9 \% & \text { SPC }=78.3 \%\end{array}$

Table 2. Averaged confusion matrix of RF-based classification on the test sets along with sensitivity (SES) and specificity (SPC).

\section{Discussion and Conclusion}

We have presented a supervised learning approach, using RF, NB and SVM classifiers, for detection of intracra- 
nial hypertension based on non- or minimally-invasive recordings of $\mathrm{ABP}$ and $\mathrm{CBFV}$. In contrast to previous works that largely relied on a single or a small set of features [3] our approach uses eighteen ABP and CBFV features. The RF classifier had the best performance in terms of sensitivity, partly due to the imbalance in the dataset, which has roughly $30 \%$ of patients with intracranial hypertension.

Limitations: We refer the reader to [13] for limitations pertaining to the data collection. Regarding the predictive power of features, we would like to issue a word of caution pertaining to all statistical evidence-based approaches. Given the closed-loop monitoring procedure in the ICU, where doctors have access to invasive ICP recordings, treatment protocols can affect correlations between ICP and ABP or CBFV features. A common intervention in intracranial hypertension is the administration of vasopressors. This, in addition to the established increase in ABP due to elevated ICP [6], could explain in part the predictive power of the feature $\mathrm{ABP}_{\text {sys }}$ (root node in Fig. 1). Along the same lines, $20 \mathrm{mmHg}$ is a clinical cutoff for treatment, and ICP is actively controlled in the ICU. This leads to a large number of recordings clustering around this value, which is detrimental to algorithmic separability and does not necessarily represent the distribution of ICP values in untreated patients.

Conclusion and Future Work: The results presented are promising. However, even though a hard cutoff on sensitivity and specificity is up for debate, significantly higher values would be required for replacing invasive ICP monitoring. Nonetheless, such an approach could be employed as a screening tool for recommending further checkups and escalation of care. The RF classifier can easily be extended to include categorical features, such as annotations about clinical interventions, the patient's medical history and lab results. Given modern multimodal brain monitoring systems, using additional measurements, such as the optic nerve sheath diameter [14], central venous pressure or near-infrared spectroscopy recordings, could uncover potential confounding factors and increase performance of the classifier further.

\section{References}

[1] M. Czosnyka and J. D. Pickard, "Monitoring and interpretation of intracranial pressure," Journal of Neurology, Neurosurgery \& Psychiatry, vol. 75, no. 6, pp. 813-821, 2004.

[2] X. Zhang, J. E. Medow, B. J. Iskandar, F. Wang, M. Shokoueinejad, J. Koueik, and J. G. Webster, "Invasive and noninvasive means of measuring intracranial pressure: a review," Physiological Measurement, vol. 38, no. 7, pp. R143-R182, 2017.

[3] D. Cardim, C. Robba, M. Bohdanowicz, J. Donnelly, B. Cabella, X. Liu, M. Cabeleira, P. Smielewski, B. Schmidt, and M. Czosnyka, "Non-invasive monitoring of intracranial pressure using transcranial Doppler ultrasonography: Is it possible?" Neurocritical Care, vol. 25, no. 3, pp. 473-491, 2016.

[4] D. Cardim, B. Schmidt, C. Robba, J. Donnelly, C. Puppo, M. Czosnyka, and P. Smielewski, "Transcranial Doppler monitoring of intracranial pressure plateau waves," Neurocritical Care, vol. 26, no. 3, p. 330, 2017.

[5] N. Carney, A. M. Totten, C. O'reilly, J. S. Ullman, G. W. Hawryluk, M. J. Bell, S. L. Bratton, R. Chesnut, O. A. Harris, N. Kissoon, et al., "Guidelines for the management of severe traumatic brain injury," Neurosurgery, vol. 80, no. 1, pp. 6-15, 2017.

[6] G. L. Clifton, M. G. Ziegler, and R. G. Grossman, "Circulating catecholamines and sympathetic activity after head injury." Neurosurgery, vol. 8, no. 1, pp. 10-14, 1981.

[7] A. F. Kalmar, J. Van Aken, J. Caemaert, E. P. Mortier, and M. M. Struys, "Value of Cushing reflex as warning sign for brain ischaemia during neuroendoscopy," British Journal of Anaesthesia, vol. 94, no. 6, pp. 791-799, 2005.

[8] M. Czosnyka, B. F. Matta, P. Smielewski, P. J. Kirkpatrick, and J. D. Pickard, "Cerebral perfusion pressure in headinjured patients: a noninvasive assessment using transcranial Doppler ultrasonography," Journal of Neurosurgery, vol. 88, no. 5, pp. 802-808, 1998.

[9] S. Jachuck, P. Ramani, F. Clark, and R. Kalbag, "Electrocardiographic abnormalities associated with raised intracranial pressure." British Medical Journal, vol. 1, no. 5952, pp. 242-244, 1975.

[10] W. Zong, T. Heldt, G. Moody, and R. Mark, "An opensource algorithm to detect onset of arterial blood pressure pulses," Computers in Cardiology, pp. 259-262, 2003.

[11] F. Wadehn, D. Carnal, and H. A. Loeliger, "Estimation of heart rate and heart rate variability from pulse oximeter recordings using localized model fitting," International Conference of the IEEE Engineering in Medicine and Biology Society, pp. 3815-3818, 2015.

[12] J. Friedman, T. Hastie, and R. Tibshirani, The Elements of Statistical Learning. Springer Series in Statistics, Berlin, 2001.

[13] F. Kashif, G. Verghese, V. Novak, M. Czosnyka, and T. Heldt, "Model-based noninvasive estimation of intracranial pressure from cerebral blood flow velocity and arterial pressure," Science Translational Medicine, vol. 4, no. 129, pp. 129ra44-129ra44, 2012.

[14] C. Robba, D. Cardim, T. Tajsic, J. Pietersen, M. Bulman, J. Donnelly, A. Lavinio, A. Gupta, D. K. Menon, P. J. Hutchinson, et al., "Ultrasound non-invasive measurement of intracranial pressure in neurointensive care: A prospective observational study," PLOS Medicine, vol. 14, no. 7, p. e1002356, 2017.

Address for correspondence:

Federico Wadehn, wadehn@isi.ee.ethz.ch, 8092 Zurich,

Switzerland 